\title{
The MSW solution to the solar neutrino problem for non-standard solar models.
}

\author{
V. Berezinsky ${ }^{(1)}$, G. Fiorentini ${ }^{(2)}$, and M. Lissia ${ }^{(3)}$ \\ (1) INFN, Laboratori Nazionali del Gran Sasso, 67010 Assergi (AQ), Italy \\ (2) Dipartimento di Fisica dell'Università di Ferrara, I-44100 Ferrara, \\ and Istituto Nazionale di Fisica Nucleare, Sezione di Ferrara, I-44100 Ferrara \\ ${ }^{(3)}$ Istituto Nazionale di Fisica Nucleare, Sezione di Cagliari, I-09128 Cagliari, \\ and Dipartimento di Fisica dell'Università di Cagliari, I-09100 Cagliari
}

(August 1994)

\begin{abstract}
The difficulties for non-standard solar models (NSSM) in resolving the solar neutrino problem are discussed stressing the incompatibility of the galliumKamiokande data, and of the gallium-chlorine data. We conclude that NSSM's cannot explain simultaneously the results of any two of the solar neutrino data (chlorine, Kamiokande and gallium). We address further the question whether the MSW solution exists for NSSM's (e.g. models with ${ }^{8} \mathrm{~B}$ neutrino flux much lower than the standard one and/or central temperature $T_{c}$ very different from $\left.T_{c}^{\mathrm{SSM}}\right)$. We demonstrate that the MSW solution exists and is very stable relative to changes of $S_{17}$ ( $S$-factor for $p+{ }^{7}$ Be reaction) and $T_{c}$. In particular, $\Delta m^{2}$ is almost constant, while $\sin ^{2} 2 \theta$ depends on the exact values of $S_{17}$ (or ${ }^{8} \mathrm{~B}$-neutrino flux) and $T_{c}$.
\end{abstract}

96.60.Kx, $14.60 \mathrm{Pq}$ 


\section{INTRODUCTION}

The standard solar model (SSM) gives a very good description of the Sun. An impressive confirmation of the SSM is given by helioseismological observations, which, according to Ref. [1], agree with the SSM predictions at the level of $0.5 \%$ for distances down to $0.2 R_{\odot}$. As a matter of fact there are at least 12 SSM's whose predictions are in reasonable agreement [2-13]. All these models include the same physics, and the slight differences between their results are mostly caused by differences in the input parameters.

However, all SSM's predict neutrino fluxes which are in disagreement with the observations of all four neutrino experiments [14 18] (see Table [1). This deficit of the detected solar neutrinos is referred to as the solar neutrino problem (SNP).

The SSM has uncertainties that basically reflect the uncertainties in the input parameters. It has been shown [19 22] that the most important uncertainties in the neutrino fluxes can be described by three parameters, the central temperature $T_{c}$, the $S$-factor $S_{17}$ for the ${ }^{7} \mathrm{Be}+p \rightarrow{ }^{8} \mathrm{~B}+\gamma$ cross section, and the ratio $S_{33}^{2} / S_{34}$ of the $S$-factors for the reactions ${ }^{3} \mathrm{He}+{ }^{3} \mathrm{He} \rightarrow{ }^{4} \mathrm{He}+2 p$ and ${ }^{3} \mathrm{He}+{ }^{4} \mathrm{He} \rightarrow{ }^{7} \mathrm{Be}+\gamma$. The uncertainty in the central temperature actually sums up uncertainties in the astrophysical factor $S_{11}$ for the $p+p \rightarrow^{2} \mathrm{He}+e^{+}+\nu$ cross section, in the solar opacity, which depends in particular on the metal abundances $Z / X$ and on possible collective plasma effects [23], in age of the Sun, and in some other quantities.

One can distinguish between SSM's and NSSM's. All SSM's consider the same physical processes, and use similar input parameters. On the contrary, NSSM's consider large changes of the input parameters, often outside their estimated uncertainties [20], and/or introduce new physical processes. In practice we assume that SSM's are characterized by the input parameters and their uncertainties as given in Ref. [20], which basically follows Bahcall [7],24].

Recently two new developments have attracted a great deal of attention. If confirmed, they can change dramatically the prediction for the solar neutrino fluxes.

The first one is the measurement [25] of $S_{17}$ factor from the cross section of dissociation for the ${ }^{8} \mathrm{~B}$ nuclei in the Coulomb field of ${ }^{208} \mathrm{~Pb}:{ }^{8} \mathrm{~B}+{ }^{208} \mathrm{~Pb} \rightarrow{ }^{208} \mathrm{~Pb}+{ }^{7} \mathrm{Be}+p$. The preliminary result gives $S_{17}=16.7 \pm 3.2 \mathrm{eV}$ barn, which should be compared with the value $S_{17}=24 \pm 2 \mathrm{eV}$ barn used by Bahcall and Pinsonneault [7]. This new result would imply a proportional reduction of the predicted ${ }^{8} \mathrm{~B}$ neutrino flux, which would come close to the flux measured by Kamiokande.

The second new development consists in the theoretical consideration of collective plasma effects in the Sun. These effects have the potentiality of lowering the solar opacity by as much as $18 \%$ [23], with the consequent lowering of the theoretical prediction of the Sun central temperature by $2 \div 3 \%$.

The previous considerations have motivated us to study NSSM's where both the astrophysical factor $S_{17}$ and the Sun central temperature are left almost as free parameters. As discussed in the next Section, this freedom, and even more so the aforementioned effects, are insufficient to resolve the SNP. It is possible to explain the observed ${ }^{8} \mathrm{~B}$ neutrino flux, i.e. the Kamiokande data, but it is not possible satisfy any two of the three experimental data (chlorine, Kamiokande and gallium) simultaneously. The basic reason for this failure is that the SNP affects now also the ${ }^{7} \mathrm{Be}$ neutrinos, and not only the rare ${ }^{8} \mathrm{~B}$ neutrinos [20,26,27]: the combination of the two gallium experiments with Homestake or Kamiokande, or the com- 
bination of Homestake with Kamiokande, implies a too low ${ }^{7}$ Be-neutrino flux. As concluded in Sect. II, the astrophysical solution is strongly disfavored.

The MSW mechanism 28,29] offers the attractive possibility of selective suppression of the ${ }^{7} \mathrm{Be}$ neutrinos, and reconciling the SSM with experiments. After having examined the input parameter uncertainties and the NSSM's as possible solutions to the SNP, it is only fair to examine how they affect the MSW solution. In particular, we ask whether the MSW solution exists for values of $S_{17}$ and central temperature significantly different from the standard ones (the range of $S_{17}$ considered includes the new preliminary result: $S_{17}=11 \div 23 \mathrm{eV}$ barn [25]).

In Section [II, we shall demonstrate the validity of the MSW solution and its weak dependence on the input parameters. We find that considerable changes of the parameters $S_{17}$ and $T_{c}$ affect the mixing angle, but $\Delta m^{2}$ remains practically the same.

\section{NUCLEAR/ASTROPHYSICAL SOLUTIONS ARE DISFAVORED}

At present the result of any (non-standard) solar model calculation, also including uncertainties in the nuclear cross sections, is inconsistent not only with the combination of the chlorine and Kamiokande data [30,31,19,21,32, but also with the combinations of the gallium data (GALLEX [17] and SAGE [18]) with either the Kamiokande [16] or chlorine [14.15] data. For a summary of the experimental data see Table $\mathbb{1}$.

The physical problem is the ${ }^{7} \mathrm{Be} /{ }^{8} \mathrm{~B}$ neutrino ratio. If both the chlorine and Kamiokande data are correct within one standard deviation, the ${ }^{7} \mathrm{Be}$ flux is negative. If we allow both experiments to go two standard deviations away from their central value, the ${ }^{7} \mathrm{Be}$ flux must still be smaller than $1.85 \times 10^{9} \mathrm{~cm}^{-2} \mathrm{sec}^{-1}$, i.e. less than $40 \%$ of its SSM value.

A model-independent analysis along the lines of Ref. [33], which only uses the luminosity constraint, shows that the combination of the present data from the gallium experiments and from Kamiokande also requires that the ${ }^{7}$ Be flux be smaller than $1.9 \times 10^{9} \mathrm{~cm}^{-2} \mathrm{sec}^{-1}$; larger values can be obtained only going more than two standard deviations from both data. These conclusions can be also derived from the comparison of the gallium and chlorine data. A similar result was recently obtained by Bahcall [27].

Nowadays the SNP is the problem of beryllium neutrinos. The low ${ }^{7} \mathrm{Be}$ flux, implied by the experimental data, is eventually the reason astrophysical solutions fail and, as we shall see, the MSW solution is stable in mass.

At the $2 \sigma$ level, any two of the chlorine, Kamiokande and gallium data implies for all existing SSM's that:

$$
\frac{\Phi\left({ }^{7} \mathrm{Be}\right)}{\Phi^{\mathrm{SSM}}\left({ }^{7} \mathrm{Be}\right)}<0.4
$$

The diminishing of the astrophysical $S_{17}$ factor to the value $15-17 \mathrm{keV}$ barn can solve the boron neutrino problem, but the flux of ${ }^{7} \mathrm{Be}$ neutrino remains too large for both the chlorine and gallium experiments.

In principle, we cannot exclude a strong suppression of ${ }^{7} \mathrm{Be} /{ }^{8} \mathrm{~B}$ ratio by nuclear physics effects, but we are able to obtain this effect only at the price of large and correlated change of parameters, which eventually are unrealistic. Let us give two examples. 
Increasing the $S_{33}$ factor we can make both fluxes, ${ }^{7} \mathrm{Be}$ and ${ }^{8} \mathrm{~B}$, very low. However, in Ref. [33] it was demonstrated that increasing $S_{33}$ by factor 11.0 still gives a poor fit to the observational data. To overcome this difficulty we can increase the proton-capture rate on beryllium $\lambda_{p}$, and/or decrease the electron capture rate $\lambda_{e}$. In this manner we can increase the production of ${ }^{8} \mathrm{~B}$ neutrinos proportionally to $\frac{\lambda_{p}}{\left(\lambda_{p}+\lambda_{e}\right)}$ (therefore compensating for the too large reduction caused by the increase of $S_{33}$ ), and decrease the ${ }^{7} \mathrm{Be} /{ }^{8} \mathrm{~B}$ ratio as $\lambda_{e} / \lambda_{p}$. However, this game works only with a rather unrealistic combination of large correlated changes of two $\left(S_{33}\right.$ and $\left.\lambda_{e}\right)$ or three $\left(S_{33}, \lambda_{e}\right.$ and $\left.\lambda_{p}\right)$ parameters.

In Ref. [20] solar models with very generous changes of the input parameters are studied. These input parameters include the central temperature $T_{c}$ (which - we recall - is determined by heavy element abundances, $S_{11}$, solar age, new plasma effects, etc.), $S_{17}$ and $S_{34}^{2} / S_{33}$. There it is demonstrated that arbitrary variations of any single one of these parameters are insufficient to explain the data of all four experiments.

We can add to this demonstration the following proof of the impossibility of an astrophysical solution where we vary the central temperature, with the aim of suppressing the ${ }^{7}$ Be neutrino flux, and, at the same time, let the astrophysical factor $S_{17}$ be a free parameter, with the hope of fixing the ${ }^{8} \mathrm{~B}$ neutrino flux.

The basic point is that the scale laws for the fluxes are such that central temperature and/or $S_{17}$ variations yield:

$$
\begin{aligned}
\frac{\Phi\left({ }^{7} \mathrm{Be}\right)}{\Phi^{\mathrm{SSM}}\left({ }^{7} \mathrm{Be}\right)} \approx\left(\frac{T_{c}}{T_{c}^{\mathrm{SSM}}}\right)^{9 \div 11} \\
\frac{\Phi\left({ }^{8} \mathrm{~B}\right)}{\Phi^{\mathrm{SSM}}\left({ }^{8} \mathrm{~B}\right)} \approx \frac{S_{17}}{S_{17}^{\mathrm{SSM}}}\left[\frac{\Phi\left({ }^{7} \mathrm{Be}\right)}{\Phi^{\mathrm{SSM}}\left({ }^{7} \mathrm{Be}\right)}\right]^{2} .
\end{aligned}
$$

In fact the ${ }^{8} \mathrm{~B}$ production is linearly proportional to $S_{17}$, since only a tiny fraction of the ${ }^{7}$ Be nuclei terminate through the ${ }^{8} \mathrm{~B}$ branch, and, therefore, the concentration of ${ }^{7} \mathrm{Be}$ nuclei is practically independent of $S_{17}$. The power of two in the ${ }^{7} \mathrm{Be}$ flux comes about because, while the ${ }^{7} \mathrm{Be}$ flux varies with temperature roughly as $T_{c}^{9 \div 11}$, the ${ }^{8} \mathrm{~B}$ flux goes roughly as $T_{c}^{18 \div 22}$ [20,24]. Therefore, Eqs. (11) and (2) imply that we need a central temperature reduction of about 10\%, and Eq. (3) together with the fact that Kamiokande sees at least $40 \%$ of the $\mathrm{SSM}{ }^{8} \mathrm{~B}$ flux implies that we need to increase $S_{17}$ of at least a factor 2.5 .

A more careful analysis that takes into account all three experiments confirms this result. We made a correlated $\chi^{2}$ analysis as function of the central temperature, and of the $S_{17}$ cross section. Since this analysis is an extension of the one done in Ref. [20] for only two values of $S_{17}$, we refer to it for details on the correlation matrix and the $\chi^{2}$ calculation.

Figure 10 shows the iso- $\chi^{2}$ curves for (a) all data, (b) only the gallium and Kamiokande data, and (c) only the gallium and chlorine data. We do not present the analysis of the Kamiokande and chlorine data, which just confirms the well-know incompatibility. We find the best $\chi^{2}$ for the combination of gallium and chlorine data, as already seen in Ref. [20]. But even if we consider this "best" combination, we obtain a $\chi^{2}$ that has a $2 \%$ probability of being a statistical fluctuations only for central temperatures at least $10 \%$ lower than the one predicted by the SSM, and $S_{17}$ larger than $60 \mathrm{eV}$ barn. For all other combinations of data we have not even been able of finding a $\chi^{2}$ compatible with statistical fluctuations at the level of $2 \%$ in the range of temperatures shown. 
The conclusion of these calculations is that we are not able to construct solar models that reproduce the experimental neutrino fluxes, even if we arbitrarily disregard one of the experiment. As a matter of fact most of the area in Fig. 1 does not correspond to NSSM's, as the ones in Ref. [20], but to truly unrealistic models. In the example where we dial both central temperature and $S_{17}$, for instance, the cross section $S_{17}$ needs to be much too large compared with its experimental determinations 34 36,25. Moreover, we obtain central temperatures for which is not even possible to construct a solar model [20] (in these cases we have used power-law extrapolations of solar model calculation).

We see, therefore, that the combined results of all four neutrino experiments are incompatible with the astrophysical solution. If one insists upon this kind of solution, one must conclude that some of the experiments are wrong. Following J. Bahcall [27] one can ask: "How many solar neutrino experiments are wrong?"

One can resolve the Homestake/Kamiokande conflict by assuming that either one is wrong. But then we are left with the conflict between the remaining experiment and the gallium experiments. Should we say that both the Kamiokande and chlorine experiments are wrong? or is the gallium data together with either Homestake or Kamiokande that is wrong?

We think that it is more reasonable to consider the non-standard neutrino option, which can explain the results of all four neutrino experiments simultaneously.

\section{THE MSW SOLUTION}

In this Section we shall study the MSW solution for NSSM's. We shall start with a semiquantitative explanation of the reason $\Delta m^{2}$ is practically the same for the large class of SSM's and NSSM's.

The MSW mechanism [28,29] suppresses the ${ }^{7}$ Be neutrinos as long as they satisfy the resonant condition in the Sun:

$$
\Delta m^{2}<\frac{2 \sqrt{2} G_{F} \rho E_{\mathrm{Be}}}{\cos 2 \theta} \approx \frac{12(\mathrm{meV})^{2}}{\cos 2 \theta} .
$$

In this equation $E_{\mathrm{Be}}=0.862 \mathrm{MeV}$ is the energy of the ${ }^{7} \mathrm{Be}$ neutrinos, and $\rho$ is approximately equal to the electron density at the peak of the production region for the ${ }^{7} \mathrm{Be}$ neutrinos (we use $89 \%$ of the central electron density).

Let us derive first the minimum $p p$ neutrino counting rate in the gallium experiments. To this end we take the $2 \sigma$ lower limit for gallium experiments, 56.4 SNU, and subtract from it the ${ }^{8} \mathrm{~B}$ and ${ }^{7} \mathrm{Be}$ signals. The ${ }^{8} \mathrm{~B}$-neutrino contribution can be found from the $2 \sigma$ upper limit of the Kamiokande counting rate. As already said in the previous Section, the combination of any two neutrino data, gives a $2 \sigma$ upper bound on the ${ }^{7} \mathrm{Be}$ neutrinos of $1.9 \times 10^{9} \mathrm{~cm}^{-2} \mathrm{sec}^{-1}$. As a result we obtain $33 \mathrm{SNU}$ as a very stringent lower limit ( $2 \sigma$ away from all three neutrino data) for the $p p$ signal in the gallium experiments. Therefore, we conclude that at least half of the $p p$ signal survives. This condition results in the lower limit for $\Delta m^{2}$ :

$$
\Delta m^{2}>\frac{2 \sqrt{2} G_{F} \rho E_{p p}}{\cos 2 \theta} \approx \frac{4(\mathrm{meV})^{2}}{\cos 2 \theta} .
$$


In this equation we use $\rho$ equal to the $67 \%$ of the central electron density, and $E_{p p}$ is defined in such way that contribution of neutrinos with energies less than $E_{p p}$ to a gallium detector is half of the total $p p$ signal. Since most of the $p p$ signal in a gallium detector is produced by neutrinos near the upper end of the energy spectrum $(0.420 \mathrm{MeV})$ we use, for the sake of discussion, $E_{p p}=0.410 \mathrm{MeV}$. Thus, if the MSW mechanism is the solution to the SNP, $\Delta m^{2}$ is bounded in a narrow interval, given by Eqs. (4) and (5), independently of the solar model we use.

When $\sin ^{2} 2 \theta<<1$, the suppression is almost complete, and the exact value of $\sin ^{2} 2 \theta$ determines only the width of the energy window where neutrinos are suppressed. For very small mixing angles, only the beryllium line is suppressed; increasing $\sin ^{2} 2 \theta$ we suppress more ${ }^{8} \mathrm{~B}$ neutrinos and/or $p p$ neutrinos (depending on the value of $\Delta m^{2}$ ). Therefore variations of astrophysical ${ }^{8} \mathrm{~B}$ flux produce only variations of the mixing angle, but leave $\Delta m^{2}$ unaffected.

Within the MSW solution the Kamiokande measurement $\Phi(\mathrm{B}) \geq 0.4 \Phi_{\mathrm{SSM}}(\mathrm{B})$, gives the minimum value which a solar model can predict. For this flux we need no suppression of the ${ }^{8} \mathrm{~B}$ neutrinos. The energy window must be very narrow, and centered on the beryllium line. The $\sin ^{2} 2 \theta$ has the smallest value. For the opposite extreme case, the maximum value of the $\Phi(\mathrm{B})$ reasonably allowed by the parameter uncertainties is about $\Phi(\mathrm{B}) \leq 1.5 \Phi_{\mathrm{SSM}}(\mathrm{B})$. This value implies a strong MSW suppression for both Kamiokande and Homestake, which in this case observe about one third of the predicted flux. Then, a large angle solution, which give almost equal suppression for energy above the resonance, becomes more favorable.

These qualitative results have been confirmed by a detailed $\chi^{2}$ analysis of the MSW solutions as function of the astrophysically produced ${ }^{8} \mathrm{~B}$ flux, and of the central temperature. The details of the calculations have already been reported in Ref. [20] for the case of the $\mathrm{SSM}^{8} \mathrm{~B}$ flux. Here we use the same procedure for the two extreme cases of minimal ${ }^{8} \mathrm{~B}$ flux compatible with Kamiokande, and the maximal ${ }^{8} \mathrm{~B}$ flux allowed by the present uncertainties, namely 2.09 and $8.7 \times 10^{6} \mathrm{~cm}^{-2} \mathrm{sec}^{-1}$, respectively. The results are shown in Fig. 2a.

In agreement with the semi-quantitative analysis given above, the reduced ${ }^{8} \mathrm{~B}$ flux allows only the small angle solution (labeled by 0.4 ), which is shifted towards smaller angles in comparison with the SSM solution (labeled by 1). In this case, we obtain a relatively large $\chi^{2}$ (5.0) for the best fit. The reason is that this reduced flux is barely compatible with the Kamiokande result. If we use a slightly larger flux, e.g. 0.5 of the SSM value, we obtain a $\chi^{2}$ similar to the other cases.

When the ${ }^{8} \mathrm{~B}$ flux increases above the SSM value, the large angle solution becomes more favorable. The allowed region becomes larger and its statistical weight increases. Simultaneously the small angle solution moves towards larger angles. For an increment described by a factor 2 (not shown in the figure), the best fit corresponds to the large angle solution.

Let us now examine the influence of the other uncertainties on the MSW solution. We parameterized these uncertainties by central temperature, which affects both ${ }^{8} \mathrm{~B}$ and ${ }^{7} \mathrm{Be}$ neutrino fluxes. One might worry that this parameterization does not cover all solar models, and does not describe the possible correlations between neutrino fluxes [37.

A priori this criticism is correct. However, several numerical studies have shown that, in practice, the effects of independent variations of the metal fraction $\mathrm{Z} / \mathrm{X}$, the opacities, the astrophysical factor $S_{p p}$, and the Sun age are well reproduced by variation of the central 
temperature of the Sun [19 22,33].

Since the ${ }^{8} \mathrm{~B}$ flux depends much more strongly on the central temperature than the ${ }^{7} \mathrm{Be}$ flux, we expect that effect of the temperature variation should be qualitatively similar to the variation of the $S_{17}$ factor. We have performed the calculation varying $T_{c}$ within the range $\pm 1 \%$, which describes the uncertainties of the SSM and within the range $\pm 2 \%$, which corresponds to NSSM's. Results are shown in Fig. 3. The MSW solution exists for all values of temperatures considered. One can see the stability of MSW especially as the mass range is concerned. The mixing angle changes slightly with temperature: the cooler Sun prefers the smaller mixing angles, while the hotter Sun revives the otherwise dying large angle solution.

We conclude this Section with an issue which has been recently raised in a paper by Gates, Krauss and White [37]. We shall show that this effect does not change our conclusions.

The definition of the confidence level (C.L.) regions is always a problem due both to our ignorance of the probability distribution, and to the different assumptions that one wants to test. Our pragmatic approach is that we feel confident about conclusions that do not depend too much on the C.L. definitions, while we feel uneasy in the opposite case. For this reason, we use in our calculations two different definitions for $95 \%$ C.L. regions.

The first definition, marked (a) in Fig. 2, answers the following question: if the MSW solution with the given parameters exists, what region of parameters has the $95 \%$ probability of containing these true parameters? The maximum likelihood procedure (with some extra assumptions) tells us that this region is given by $\chi^{2}<\chi_{\min }^{2}+\Delta \chi^{2}$, where $\chi_{\min }^{2}$ is the value of $\chi^{2}$ for the best fit, and $\Delta \chi^{2}$ depends on the number of parameters and on the C.L. For example, $\Delta \chi^{2}=5.99$ for 2 parameters and a $95 \%$ C.L. We used this definition in our previous publications [33, 38 .

The second definition (b) answers a different question: what is the region of parameters where the MSW mechanism does not contradict the experimental data at a given C.L.? Again under some additional assumptions, this region is given by $\chi^{2}<\delta \chi^{2}$, where now $\delta \chi^{2}$ depends on the number of data points and the C.L. For example, $\delta \chi^{2}=7.82$ for 3 experiments and a $95 \%$ C.L.

Note that definition (a) will always give us some confidence region, since we assume that the MSW mechanism exists, while definition (b) can yield no allowed region at all: in this case we conclude that the MSW mechanism fails to provide the given C.L. In fact, only when $\chi_{\min }^{2}$ is large (bad fit) the two definitions give qualitatively different regions: in this case we should worry about the original assumption under which (a) is valid, i.e. that the MSW solution exists.

Figure 2 shows that for our purposes the two definitions are very similar, and cannot change our conclusions. The same is true for all our calculations, and, therefore, in Fig. 3 we have used only the first definition for the confidence regions.

\section{CONCLUSIONS}

For standard massless neutrinos the data of any two solar neutrino measurements (chlorine, Kamiokande and gallium) are incompatible. The incompatibility of the chlorine and Kamiokande data is a well recognized problem [30, 31, 19, 21, 26, 32, The conflict between the gallium and Kamiokande data, and between the gallium and chlorine data, can be shown in 
different ways. One can obtain [26] a rigorous model-independent lower limit for the gallium detector counting rate by neglecting the ${ }^{7} \mathrm{Be}$ neutrino flux, using the observational lower limit for the ${ }^{8} \mathrm{~B}$ neutrino flux, and taking the flux of $p p$ neutrinos from the solar luminosity restriction. It gives $82.5 \mathrm{SNU}$ to be compared with the combined result of the two gallium experiments $74 \pm 9$ SNU. Using the gallium data, the luminosity constraint, and the lower limit for the flux of ${ }^{8} \mathrm{~B}$ neutrinos from either Homestake or Kamiokande, one can derive [20] a model-independent limit on the ${ }^{7} \mathrm{Be}$ neutrino flux. The updated limit using the new gallium data [17, 18] is $1.9 \times 10^{9} \mathrm{~cm}^{-2} \mathrm{~s}^{-1}(2 \sigma)$, i.e. less than $40 \%$ of the SSM value. Most recently J. N. Bahcall [27] found that the ${ }^{7}$ Be neutrino signal in a gallium detector is less than 19 SNU at the 95\% C.L., about half of the 36 SNU predicted by the SSM.

We have demonstrated that there are no values of $S_{17}$ and $T_{c}$, the two most important solar-model parameters as neutrino fluxes are concerned, which satisfy the combination of any two experimental data out of three (chlorine, Kamiokande and gallium).

Nowadays, the essence of solar neutrino problem is the low ${ }^{7} \mathrm{Be}$ neutrino flux. Nuclear/astrophysical solutions to the solar neutrino problem are strongly disfavored. The MSW mechanism offers a very attractive solution to the solar neutrino problem.

The new measurements of $S_{17}$ factor [25] and collective plasma effects [23] can considerably change the solar-model parameters $S_{17}$ and $T_{c}$. In fact, we might face what now are non-standard solar models, which are also incompatible with the solar neutrino data. Does the MSW solution exist for these models?

We have demonstrated that the MSW solution exists for NSSM's with parameters $S_{17}$ and $T_{c}$ within their realistic uncertainties, and beyond. Our conclusion concerning the ${ }^{8} \mathrm{~B}$ neutrino flux coincides with the results of Ref. [39]. The MSW solution is stable in mass $\left(\Delta m^{2}\right.$ ) even if $S_{17}$ or $T_{c}$ are drastically changed (a change of $S_{17}$ practically results in only a proportional change of the ${ }^{8} \mathrm{~B}$ neutrino flux). In particular, $\Delta m^{2}$ is restricted to the range $4(\mathrm{meV})^{2}<\Delta m^{2}<12(\mathrm{meV})^{2}$. The physical reason of this stability is the fact that the solar neutrino experiments observe most of the predicted flux of $p p$ neutrinos, and very small fraction of the ${ }^{7}$ Be neutrino flux.

Solar-model predictions for the ${ }^{8} \mathrm{~B}$ neutrino flux are only important for the determination of the mixing angle. Models with reduced ${ }^{8} \mathrm{~B}$ neutrino flux prefer the small angle solution, because of the stronger suppression of the ${ }^{7} \mathrm{Be} /{ }^{8} \mathrm{~B}$ flux ratio. The large angle solution disappears for models with reduced ${ }^{8} \mathrm{~B}$ neutrino flux. Large-mixing-angle solutions reappear in models with hotter core and/or larger $S_{17}$ factor, since they predict a ${ }^{8} \mathrm{~B}$-neutrino-flux increase larger than the corresponding increase of the ${ }^{7}$ Be flux.

\section{ACKNOWLEDGMENTS}

One of us (ML) wishes to thank the Organizers of the "Summer Institute on Nuclear Physics and Astrophysics: Prospects for Underground Research" held at Laboratori Nazionali del Gran Sasso, Italy, in June and July 1994, where part of this work was done. 


\section{NOTE ADDED}

While this paper was in preparation we received two preprints [27,39 that are relevant to our work.

J. N. Bahcall [27] studies the incompatibility of the gallium data with either Homestake or Kamiokande. Our method is essentially different from his, but our conclusions coincide. This same incompatibility was also discussed in Refs. 20] and [26].

P. I. Krastev and A. Yu. Smirnov [39] obtain some of our same results. In particular, these authors notice the mass stability of the MSW solution relative to variation of ${ }^{8} \mathrm{~B}$ neutrino flux. Numerically, our results agree with theirs. 


\section{REFERENCES}

[1] S. Turck-Chièze et al., Phys. Rep. 230 (1993) 57.

[2] J. N. Bahcall and R. K. Ulrich, Rev. Mod. Phys. 60 (1988) 297.

[3] Y. Lebreton and W. Däppen, in: Seismology of the Sun and the Sun-like Stars, eds. V. Domingo and E. J. Rolfe (European Space Agency, Nordwijk, 1988).

[4] I. J. Sackman, A. I. Boothroyd and W. A. Fowler, Ap. J. 360 (1990) 727.

[5] C. R. Proffitt and A. N. Cox, Ap. J. 380 (1991) 238.

[6] J. A. Guzik and A. N. Cox, Ap. J. 381 (1991) 331.

[7] J. N. Bahcall and M. H. Pinsonneault, Rev. Mod. Phys. 64 (1992) 885.

[8] D. B. Günter et al., Ap. J. 387 (1992) 372.

[9] J. Chistiansen-Dalsgaard, Geophys. Astrophys. Fluid. Dyn. 62 (1992) 123.

[10] B. Ahrens, M. Stix and M. Thorn, A.\& A. 264 (1992) 673.

[11] G. Bartomieu, J. Provost and J. Morel, A.\& A. 268 (1993) 775.

[12] S. Turck-Chièze and I. Lopes, Astrophys. J. 408 (1993) 347.

[13] V. Castellani, S. Degl'Innocenti and G. Fiorentini, A.\& A. 271 (1993) 601.

[14] R. Davis Jr., in: Proc. of the 23rd ICRC (Calgary, Canada 1993), Prog. in Nucl. and Part. Phys. 32 (1994).

[15] R. Davis Jr., Report on the Homestake solar neutrino experiment, in: Proc. of the International Topical Workshop on "Solar Neutrino Problem: Astrophysics or Oscillations?" (Assergi, Italy, February 1994), Vol. 1, eds. V. Berezinsky and E. Fiorini (LNGS, 1994) p. 66.

[16] A. Suzuki, Kamiokande results and prospects, in: Proc. of the 6th International Symposium on Neutrino Telescopes (Venice, February 1993), ed. M. Baldo Ceolin.

[17] GALLEX Collaboration, P. Anselman et al., Phys. Lett. B 327 (1994) 377.

[18] SAGE collaboration, J. N. Abdurashitiv et al., in: Proc. of the 27th Int. Conf. on High Energy Physics (Glasgow, July 1994).

[19] V. Castellani, S. Degl'Innocenti and G. Fiorentini, Phys. Lett. B 303 (1993) 68.

[20] V. Castellani et al., Ferrara Preprint INFNFE-3-94 (1994), to appear in: Phys. Rev. D.

[21] N. Hata, S. A. Bludman and P. Langacker, Phys. Rev. D 49 (1994) 3622.

[22] N. Hata, University of Pennsylvania Preprint UPR-0612T (1994).

[23] V. Tsytovich, Collective plasma effects in the radiative transport in solar interior, in: Proc. of the International Topical Workshop on "Solar Neutrino Problem: Astrophysics or Oscillations?" (Assergi, Italy, February 1994), Vol. 1, eds. V. Berezinsky and E. Fiorini (LNGS, 1994) p. 238.

[24] J. N. Bahcall, Neutrino Astrophysics (Cambridge University Press, Cambridge, 1989).

[25] T. Motobayashi et al., Rikkyo Preprint RUP-94-2 (1994).

[26] V. Berezinsky, INFN LNGS Preprint LNGS-94/101, to appear in: Proc. of the 7th International Symposium on Neutrino Telescopes (Venice, February 1994), ed. M. Baldo Ceolin.

[27] J. N. Bahcall, Princeton IAS Preprint IASSNS-AST-94-37 (1994).

[28] L. Wolfenstein, Phys. Rev. D 17, (1978) 2369.

[29] S. P. Mikheyev and A. Yu. Smirnov, Nuovo Cimento C 9 (1986) 17.

[30] J. N. Bahcall and H. A. Bethe, Phys. Rev. Lett. 65 (1990) 2233.

[31] J. N. Bahcall and H. A. Bethe, Phys. Rev. D 47 (1993) 1298. 
[32] V. Berezinsky, INFN LNGS Preprint LNGS-93/86 (1993), to appear in: Comments on Nuclear and Particle Physics.

[33] V. Castellani et al., Phys. Lett. B 324 (1994) 245.

[34] R. W. Kavanagh et al., Bull. Am. Phys. Soc. 14 (1969) 1209.

[35] B. W. Filippone et al., Phys. Rev. C 28 (1983) 2222.

[36] K. Langanke and T. D. Shoppa, Cal Tech Kellogg Lab Preprint MAP-168 (1994).

[37] E. Gates, L. M. Krauss and M. White, Fermilab Preprint FERMILAB-PUB-94-176-A (1994).

[38] G. Fiorentini et al., Phys. Rev. D 49 (1994) 6298.

[39] P. I. Krastev and A. Yu. Smirnov, Preprint DOE-ER-40561-137 and INT94-13-02 (1994). 


\section{FIGURES}

FIG. 1. Contours of equal $\chi^{2}$ for the neutrino fluxes in solar models parameterized by the central temperature (normalized to its SSM value), and by the $S_{17}$ astrophysical factor. Full contours correspond to $\chi^{2}$ equal to 40,30, 20 and 10; dashed contours correspond to $\chi^{2}$ equal to $35,25,15$ and 5. Values of $\chi^{2}>9.84$ have a $2 \%$ probability for three data (a), i.e. for all experiments (the two gallium data have been combined). Cases (b) and (c) show $\chi^{2}$ for two experimental data: gallium and Kamiokande (b) and gallium and chlorine (c). In this case values of $\chi^{2}>7.82$ have a $2 \%$ probability. Graphs (b) and (c) show the contradiction between the gallium results, and either of two other experiments (chlorine or Kamiokande): even a $\chi^{2}<10$ (less than $1 \%$ probability for values larger than 10) needs too high values of $S_{17}$.

FIG. 2. Regions of the MSW solution allowed at the 95\% C.L. The region labeled 1 corresponds to the SSM [13] with the standard ${ }^{8} \mathrm{~B}$ neutrino flux; in the region labeled $0.4(1.5)$ the ${ }^{8} \mathrm{~B}$ flux is reduced (increased) by a factor 0.4 (1.5). Note that the large angle solution disappears when the flux is reduced (0.4). The filled black circles show the best fit for each flux: the corresponding $\chi_{\min }^{2}$ are 5.0, 0.22 and 0.32 , for flux factors $0.4,1$ and 1.5, respectively. The confidence regions are defined as (a) $\chi^{2}=\chi_{\min }^{2}+5.99$, and (b) $\chi^{2}=7.82$ (see text).

FIG. 3. Regions of the MSW solutions allowed at the 95\% C.L. for models with varied central temperature. In (a) the central temperature $T_{c}$ is $\pm 1 \%$ of $T_{c}^{\mathrm{SSM}}$, the corresponding temperature in the SSM [13]. Note that the large angle MSW solution disappears when the temperature is reduced. In (b) the central temperature is $\pm 2 \%$ of $T_{c}^{\mathrm{SSM}}$ (these are already NSSM's). There is again no large angle solution when the temperature is reduced. In both figures the black filled circles give the best fit: the corresponding $\chi_{\min }^{2}$ are $1.38,0.76,0.22,0.12$ and 0.45 , respectively for temperature changes of $-2 \%,-1 \%$, no change (SSM), $+1 \%$ and $+2 \%$. The confidence regions are defined as $\chi^{2}=\chi_{\min }^{2}+5.99$ (see text). 


\section{TABLES}

TABLE I. Most recent experimental data (Experiment), and a selected sample of theoretical predictions: Bahcall and Pinsonneault [7] (BP SM), Turck-Chièze et al. [12] (TCL SM) and Castellani et al. [33] (CDF SM). In this work we combine systematic and statistical errors of the experimental data and the GALLEX and SAGE data, and use a slightly different chlorine data (Data used). The errors of the theoretical works are $1 \sigma$ effective errors.

\begin{tabular}{|c|c|c|c|c|}
\hline & \multirow{2}{*}{$\begin{array}{l}\text { Chlorine } \\
\text { (SNU) }\end{array}$} & \multirow{2}{*}{$\begin{array}{c}\text { Kamiokande } \\
\left(10^{6} \mathrm{~cm}^{-2} \mathrm{~s}^{-1}\right)\end{array}$} & \multicolumn{2}{|c|}{ Gallium (SNU) } \\
\hline & & & GALLEX & SAGE \\
\hline Experiment & $2.32 \pm 0.23^{\mathrm{a}}$ & $2.90 \pm 0.23 \pm 0.35^{\mathrm{b}}$ & $79 \pm 10 \pm 6^{\mathrm{c}}$ & $69 \pm 11 \pm 6^{\mathrm{d}}$ \\
\hline Data used & $2.28 \pm 0.23^{\mathrm{e}}$ & $2.90 \pm 0.42$ & \multicolumn{2}{|c|}{$74.4 \pm 8.8$} \\
\hline $\mathrm{BP} \mathrm{SM}$ & $8.0 \pm 1.0$ & $5.69 \pm 0.80$ & \multicolumn{2}{|c|}{$132_{-6}^{+7}$} \\
\hline TCL SM & $6.4 \pm 1.4$ & $4.4 \pm 1.1$ & \multicolumn{2}{|c|}{$123 \pm 7$} \\
\hline CDF SM & $7.8 \pm 1.0$ & $5.6 \pm 0.8$ & \multicolumn{2}{|c|}{$130 \pm 6$} \\
\hline
\end{tabular}

${ }^{\text {aRef. [15] }}$

${ }^{b}$ Ref. [16]

${ }^{\mathrm{c}}$ Ref. 17

${ }^{\mathrm{d}}$ Ref. 18

${ }^{\mathrm{e}}$ Ref. 14. 
This figure "fig1-1.png" is available in "png" format from: http://arxiv.org/ps/hep-ph/9408304v2 


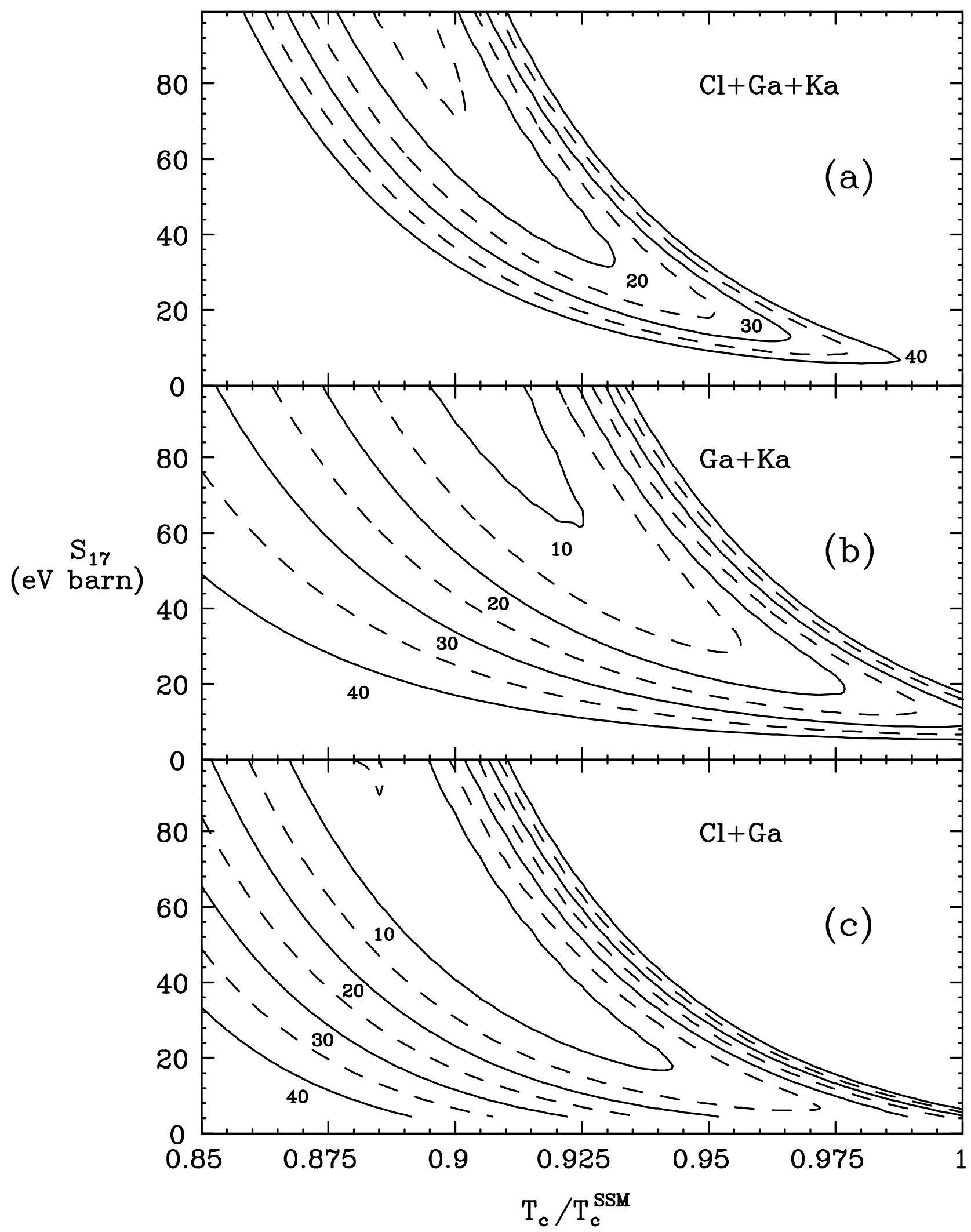

Fig. 1

Berezinky et al., The MSW solution ....

LGNS-94-104, Sub. to PLB August 1994 
This figure "fig1-2.png" is available in "png" format from: http://arxiv.org/ps/hep-ph/9408304v2 


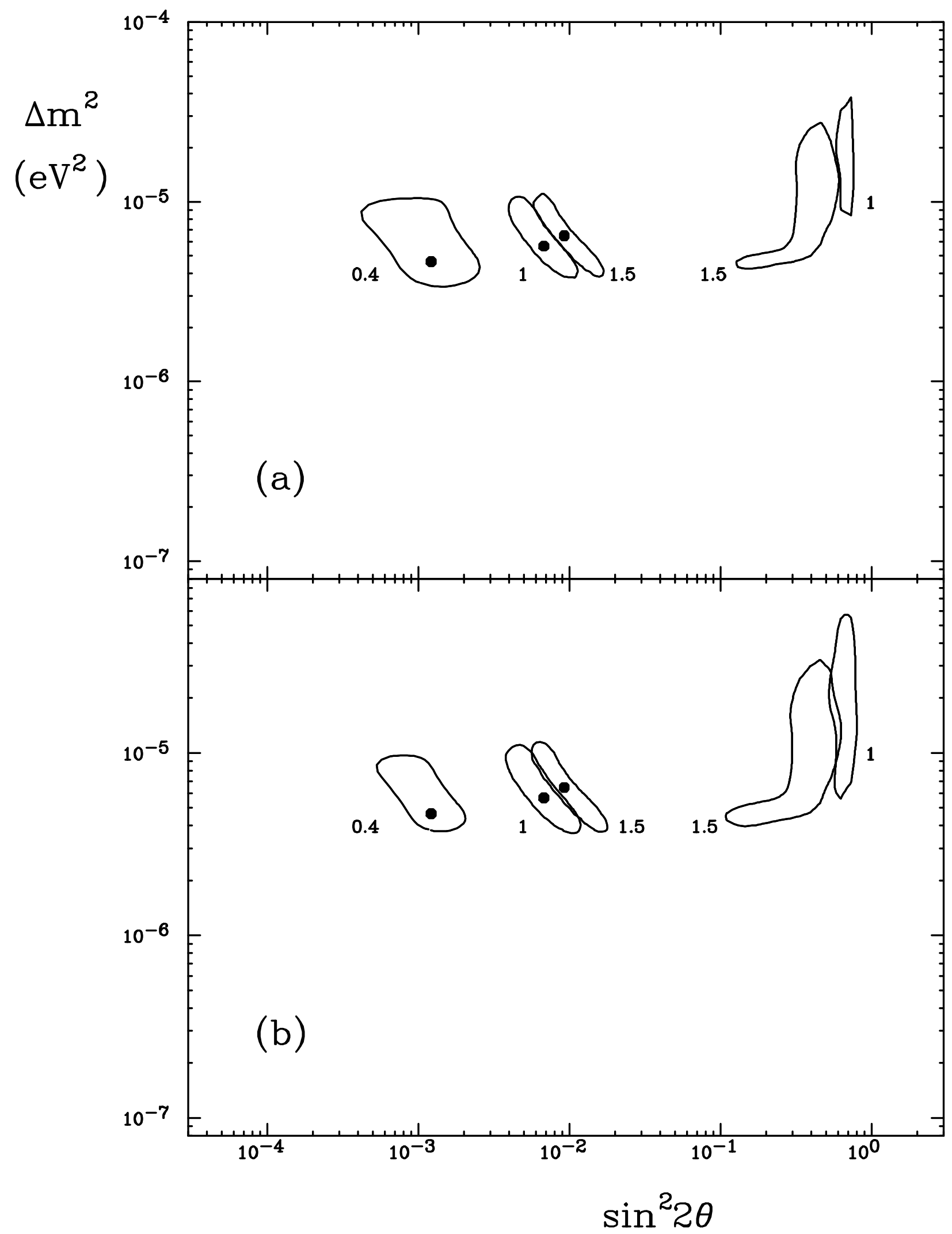

Fig. 2

Berezinky et al., The MSW solution man

LGNS-94-104. Sub. to PLB Auaust 1994 
This figure "fig1-3.png" is available in "png" format from: http://arxiv.org/ps/hep-ph/9408304v2 


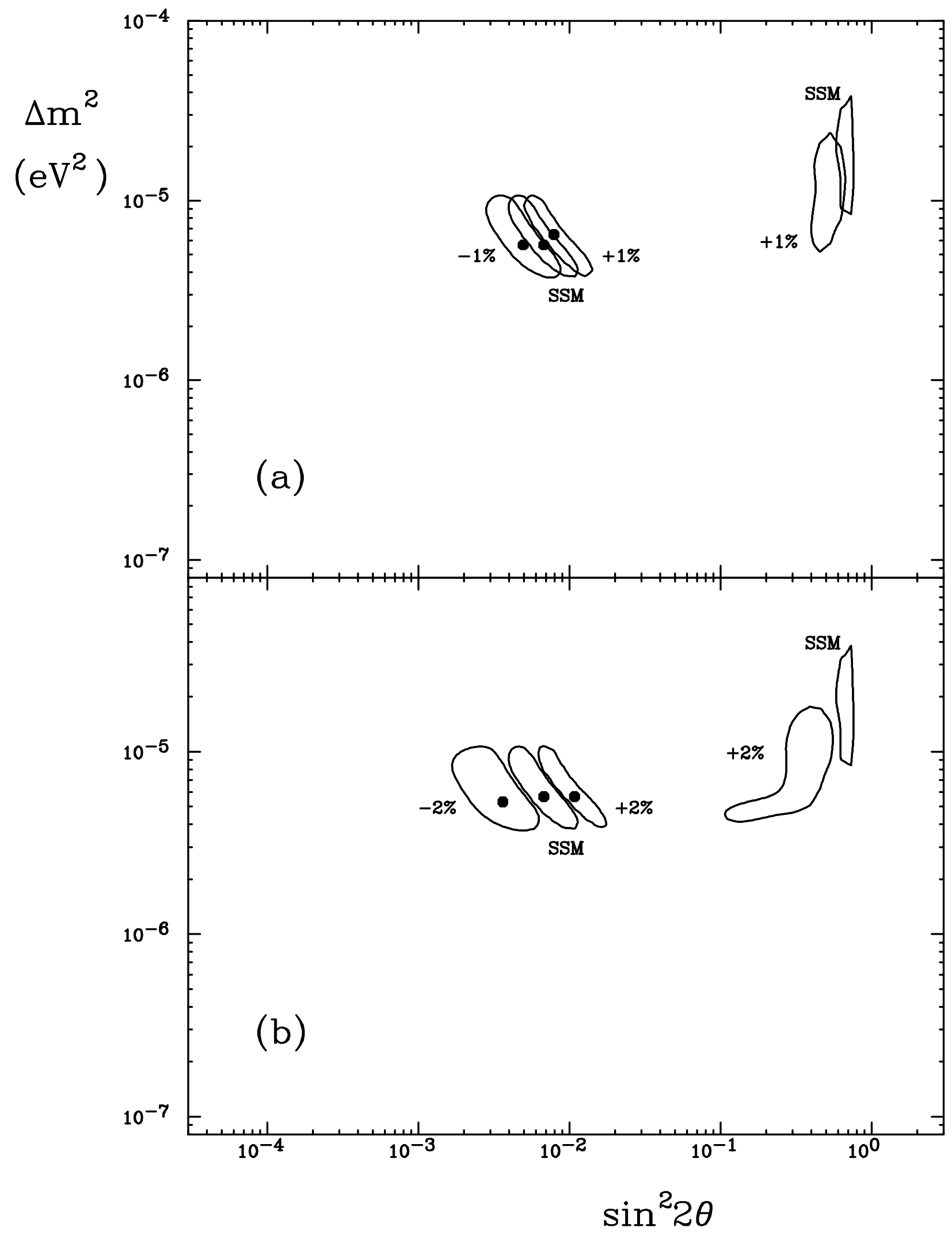

Fig. 3

Berezinky et al., The MSW solution ang

LGNS-94-104. Sub. to PLB Auaust 1994 